\title{
Forschungsnotiz
}

\section{Die Verläßlichkeit von Angaben acht- bis zehnjähriger Kinder über den Beruf des Vaters}

\section{Sabine Lang \\ Sigrid Breuer}

Sonderforschungsbereich 3, Universität Mannheim, Tattersallstr. 2 (Rheinhof), D-6800 Mannheim

\begin{abstract}
Zusammenfassung: Anhand von Daten einer im Sonderforschungsbereich 3 durchgeführten Kinderbefragung wird die Zuverlässigkeit der Angaben von Kindern über den Beruf des Vaters überprüft, indem Kinder- und Elternangaben hierzu verglichen werden. Die Ergebnisse zeigen, daß von über 1000 Kindern nur 36 Kinder wirklich fehlerhafte Angaben machen. Die Angaben von 170 Kindern sind nicht eindeutig als richtig oder falsch zu klassifizieren. Der allgemeine Schluß, den wir daraus ziehen, ist, daß man Kinder im Grundschulalter zu objektiven Sachverhalten befragen und mit zuverlässigen Antworten rechnen kann.
\end{abstract}

\section{Einleitung}

Die Befragung von Kindern über ihre Lebensbedingungen, ihre Einstellungen und ihr Verhalten ist in der Umfrageforschung bislang nicht üblich gewesen. In jüngster Zeit mehren sich jedoch die Stimmen, teilweise im Zusammenhang mit der Forderung nach einer Sozialpolitik für Kinder (Kaufmann/Lüscher 1979), die dafür plädieren, Kinder, auch solche im Grundschulalter, selbst zu befragen, wenn es um Themen geht, die Kinder betreffen (Lüscher 1977). Gleichzeitig mit dieser Forderung werden allerdings auch kritische Stimmen laut, die auf die Schwierigkeiten eines solchen Unterfangens hinweisen (Götte 1979). Implizit wird den Kindern die Unfähigkeit unterstellt, richtige Auskünfte über objektive Sachverhalte ihres Lebens zu geben.

Empirische Untersuchungen, die sich mit der Übereinstimmung von Kinder- und Elternangaben zu sozio-demographischen Merkmalen der Familie beschäftigen, kommen dabei zu unterschiedlichen Ergebnissen. Während in verschiedenen Studien nur mäßige Übereinstimmungen zwischen Kinderund Elternangaben gefunden werden (Hess/Torney 1967; Saint John 1970; Bauer 1984), berichten andere über sehr viel positivere Erfahrungen bei der Befragung von Kindern (Cohen/Orum 1972; Meulemann/Wieken-Mayser 1984).

Der Frage, inwieweit Kinder im Grundschulalter in der Lage sind, zuverlässige Auskünfte über bestimmte sozio-demographische Merkmale ihrer Familie zu geben, wird im folgenden anhand eigener empirischer Ergebnisse nachgegangen.
Die berichteten Daten stammen aus einer im Jahr 1980 durchgeführten bundesweiten, repräsentativen Kinderbefragung, die Aufschluß über die Lebensbedingungen und die wahrgenommene Lebensqualität acht- bis zehnjähriger Grundschulkinder geben sollte (,Kindersurvey 1980“). Es wurden 2048 Kinder der zweiten bis vierten Grundschulklassen in ihren Klassenverbänden mit einem standardisierten Fragebogen $\mathrm{zu}$ den Lebensbereichen „Familie“, „Schule“, „Spiel“, „Wohnsituation", „soziale Kontakte“ sowie zu ihren Bedürfnissen, Wünschen, Ängsten und Sorgen befragt. Eine zusätzliche schriftliche Elternbefragung ( $N$ = 1284) über Erziehungsziele und -stile, Lebensbedingungen, Erwartungen und Einstellungen der Eltern erbrachte zusätzliche Informationen und ermöglichte darüber hinaus Vergleiche zwischen Kinder- und Elternangaben zu einer Reihe objektiver Sachverhalte. Ein Merkmal, das bei Kindern und Eltern erfaßt wurde, ist der Beruf des Vaters. Anhand der Daten des Kindersurvey 1980 soll geprüft werden, inwieweit Kinder- und Elternangaben zum Beruf des Vaters übereinstimmen und ob abweichende Angaben möglicherweise zu erklären sind. Für diese Auswertungen konnten 1096 Eltern-Kind-Paare herangezogen werden.

Die Vercodung der von Kindern und Eltern genannten Berufsbezeichnungen erfolgte nach der „ISCO“ (Internationale Standardklassifikation der Berufe). „ISCO“ ist eine Berufsklassifikation nach acht Berufshauptgruppen und 83 Untergruppen, die sich wiederum in 284 Berufsgattungen aufteilen. Für die Haupt- und Untergruppen liegen Tätigkeitsbeschreibungen vor, die eine Klassifikation 
auch dann ermöglichen, wenn die Befragten statt der Nennung der Berufsnamen lediglich Tätigkeitsbeschreibungen abgeben. Eine Vercodung nach dieser differenzierten Klassifikation erlaubt es, die Angaben der Befragten bei verhältnismäßig geringem Informationsverlust maschinell auszuwerten. Eine Bewertung der Zuverlässigkeit der Angaben von Kindern über den Beruf des Vaters erfordert jedoch im Zweifelsfall einen Vergleich der wörtlichen Nennungen von Kindern und Eltern. Deshalb wird im folgenden größtenteils auf die individuellen Antworten und nicht auf die vercodeten Angaben eingegangen.

\section{Die Übereinstimmung von Berufsangaben bei Kindern und Eltern}

Einen ersten Aufschluß über das Ausmaß der Übereinstimmung erhält man bereits bei der Gegenüberstellung der vercodeten Berufsangaben von Kindern und Eltern. Dabei zeigt sich, daß nur eine knappe Mehrheit (52\%) der Kinderangaben zum Beruf des Vaters mit den Elternangaben übereinstimmt.

Die Übereinstimmung zwischen Kindern und Eltern erweist sich als unabhängig vom Alter der Kinder, von ihrer Stellung in der Geschwisterreihe und von der Sozialschicht, der die Familie angehört. Dagegen hat die Schulleistung der Kinder einen Einfluß auf ihre Fähigkeit, den Beruf des Vaters richtig anzugeben. Darüber hinaus spielt auch der Beruf selbst eine Rolle dabei, ob die
Kinder richtige Angaben machen. Arbeiterberufe und handwerkliche Berufe werden ebenso wie Berufe aus der Landwirtschaft von den Kindern häufiger korrekt berichtet als technische oder wissenschaftliche Berufe, Handelsberufe oder leitende Tätigkeiten in der Wirtschaft oder im öffentlichen Dienst.

\section{Abweichende Angaben}

Untersucht man die Abweichungen anhand der wörtlichen Angaben der Befragten, lassen sich für den überwiegenden Teil nicht-übereinstimmender Angaben Erklärungen finden. In einem weiteren Teil der Fälle sind die Antworten nicht eindeutig als richtig oder falsch einzustufen und lassen somit keine Rückschlüsse auf die Auskunftsfähigkeit der Kinder zu. Nur eine geringe Anzahl voneinander abweichender Kinder- und Elternangaben läßt sich nicht erklären. Lediglich in diesen Fällen erscheint es gerechtfertigt, davon auszugehen, daß die Kinder nicht in der Lage sind, den Beruf des Vaters korrekt zu benennen. Im folgenden soll auf diese drei Kategorien von Abweichungen näher eingegangen werden.

\section{a) Erklärbare Abweichungen}

Ein großer Teil - $61 \%$ - der unterschiedlich vercodeten Kinder- und Elternangaben kann nach einem Vergleich der wörtlichen Nennungen doch als übereinstimmend gewertet werden. Die Art dieser Abweichungen läßt sich in einigen wenigen Kate-

Tabelle 1 Ursachen für abweichende Vercodungen bei offensichtlich übereinstimmenden Kinder- und Elternangaben

26,2

22,5

Fische - selbständiger Kaufmann)

einmal Nennung des Berufsnamens, einmal Beschreibung der Tätigkeit (z. B. Elektriker repariert elektrische Geräte)

Vercodungsfehler, weil Beruf nicht in ISCO-Liste

zwei Angaben in einem Fragebogen, davon nur eine vercodet

Fehler aufgrund falscher Lehrerangabe
$\%$

14,4

11,9

38

N

46

84

72

$8,1-26$

$11,3 \quad 36$

2,58

$3,1 \quad 10$


gorien zusammenfassen: Unterschiedliche Formulierungen und eine unterschiedliche Präzision bei der Berufsangabe führten besonders häufig zu unterschiedlichen Codierungen. Daneben sind Abweichungen darauf zurückzuführen, daß die Antworten der Kinder und Eltern den Berufsnamen, eine Tätigkeitsbeschreibung oder die Angabe der arbeitsrechtlichen Stellung, der hierarchischen Position im Betrieb bzw. des Ortes der Berufsausübung in jeweils unterschiedlicher Kombination zum Inhalt hatten. Eine weitere Fehlerquelle bildeten identische, aber sehr vage Formulierungen bei Kindern und Eltern, die zu unterschiedlichen Klassifikationen führten, da diese unpräzisen Angaben in der ISCO-Liste keine direkte Entsprechung fanden. Nur selten kam es dadurch zu abweichenden Ergebnissen, daß Kinder und Eltern mehrere Angaben machten, von denen nur eine vercodet wurde. In einigen Fällen hatten die zusätzlichen Angaben der Lehrer, mit deren Hilfe die Kinderangaben überprüft wurden, zu fehlerhaften Vercodungen geführt. Tabelle 1 zeigt noch einmal, welche Ursachen in welchem Ausmaß für die Abweichungen verantwortlich sind.

\section{b) Nicht eindeutig erklärbare Abweichungen}

Von allen abweichenden Angaben waren insgesamt 170 nicht eindeutig erklärbar (32\%). Diese Abweichungen beruhten teilweise wieder auf unterschiedlichen Formulierungen $(\mathrm{N}=16)$ und einer unterschiedlichen Genauigkeit der Angaben $(N=16)$. Besonders häufig kam es vor, daß Kinder und Eltern auf verschiedenen Dimensionen antworteten $(\mathrm{N}=114)$.

Bei der Suche nach Erklärungen für die restlichen 24 abweichenden Berufsangaben wurden die einzelnen Kinder- und Elternfragebögen insgesamt noch einmal genau angesehen. Dadurch ließen sich in einer Reihe von Fällen weitere Ursachen für unterschiedliche Nennungen bzw. unterschiedliche Vercodungen finden. Zwei Kinder hatten geschiedene Mütter, die wieder geheiratet hatten. Während die Mütter den Beruf ihres derzeitigen Mannes angaben, nannten die Kinder offensichtlich den Beruf ihres leiblichen Vaters. Zwei Kinder lebten nicht bei den leiblichen Eltern, sondern bei den Großeltern bzw. bei Verwandten, die den Fragebogen für ihre Person ausgefüllt hatten, während die Kinder die Anworten auf ihre Eltern bezogen. In zwei Fällen läßt sich die Nicht-Übereinstimmung von Kinder- und Elternangaben dadurch erklären, daß die Väter zur Zeit der Befragung Umschulungsmaßnahmen mitmachten. Die
Kinder gaben hier vermutlich den ehemaligen, die Eltern dagegen den gerade neu zu erlernenden Beruf des Vaters an. In sechs Fällen war die Zuordnung von Kinder- und Elternfragebögen falsch. Dies war aus anderen, nicht-übereinstimmenden Angaben in den betreffenden Fragebögen zu ersehen. Diese falsche Zuordnung läßt sich so erklären, daß die Kinder in der Schulklasse untereinander die Briefumschläge mit den Elternfragebögen getauscht hatten. Für diese Erklärung spricht auch die Tatsache, daß es sich bei den sechs falschen Zuordnungen um drei „Paare“ handelt, die jeweils aus derselben Schulklasse stammten. In acht weiteren Fällen ist ebenfalls eine falsche Fragebogenzuordnung zu vermuten, was jedoch nicht eindeutig zu belegen ist. In vier Fällen schließlich machten die von den Eltern ausgefüllten Fragebögen einen fehlerhaften Eindruck. Viele Fragen waren offensichtlich nicht verstanden worden, die offenen Fragen waren häufig gar nicht oder fehlerhaft beantwortet und die Formulierungen ließen auf Sprachschwierigkeiten schließen. In der Tat handelt es sich hier um ausländische Familien.

\section{c) Nicht erklärbare Abweichungen}

Schließlich bleibt noch ein Rest von 36 Fällen $(7 \%)$, bei denen Kinder und Eltern voneinander abweichende Angaben zum Beruf des Vaters gemacht haben, ohne daß sich bei Durchsicht der Fragebögen hierfür eine Erklärung hätte finden lassen. Um dem Leser eine eigene Beurteilung zu ermöglichen, sind einige dieser Fälle in Tabelle 2 aufgelistet.

Tabelle 2 Voneinander abweichende Angaben von Kindern und Eltern zum Beruf des Vaters

\begin{tabular}{ll}
\hline Feuerwehr & KFZ-Schlosser \\
Automechaniker & Heizungsmonteur \\
Metallarbeiter & Schreiner \\
Fensterputzer & Elektriker \\
Bäcker & Schweißer bei VW \\
Koch & Elektrotechniker \\
Techniker & Pastor \\
Schweißer & Berater \\
Schreiner & kaufmännischer Angestellter \\
Zeitungsfahrer & Rolladenbauer \\
Kunststoffschlosser & Hausmeister \\
Schreiner & Straßenwärter
\end{tabular}

\section{Schlußbetrachtung}

Wenn man von der berücksichtigten Stichprobe von 1096 Fällen ausgeht, erscheint die Zahl von 36 
fehlerhaften Kinderangaben und 170 nicht eindeutig als richtig oder falsch zu klassifizierenden Angaben eher gering. Der Gesamteindruck, den die Ergebnisse vermitteln, bestätigt unseres Erachtens die Position derer, die davon ausgehen, daß man Kinder zu objektiven Daten der Familie befragen und dabei mit zuverlässigen Antworten rechnen kann. Allerdings scheint auch klargeworden zu sein, daß - zumindest im Fall der Berufsangaben nicht mit besonders detaillierten Auskünften zu rechnen ist. Vielmehr lassen sich die Angaben lediglich in grobe Kategorien einordnen. $\mathrm{Ob}$ es sinnvoll wäre, solche relativ groben Raster direkt vorzugeben und die Kinder nur noch aufzufordern, die Elternberufe hier einzuordnen, kann aufgrund der vorliegenden Ergebnisse nicht entschieden werden. Ein Grund, der dagegen sprechen würde, ist die Tatsache, daß die Vorgabe eines solchen Rasters sehr abstrakt ist und Kinder im Grundschulalter überfordern könnte. Allerdings erscheint es angebracht, die Frage nach dem Beruf selbst noch stärker in die eine oder andere Richtung hin zu präzisieren (arbeitsrechtliche Stellung, Tätigkeitsbeschreibung), je nachdem, an welcher Information dem Forscher gelegen ist. Durch gezieltes und systematisches Nachfragen kann die Informationsqualität verbessert werden. Bei der hier gewählten Befragungsmethode der "Classroom-Interviews" war uns ein solches „Nachhaken" nur gelegentlich und unsystematisch möglich. Allerdings haben auch solche Nachfragen ihre Grenzen, wenn die Kinder in der Tat nicht mehr über den Beruf des Vaters wissen als z. B., daß er „im Büro“ oder bei einer bestimmten Firma beschäftigt ist.

Die hier berichteten Ergebnisse lassen keine Aufschlüsse darüber zu, ob ein Erheben subjektiver Daten wie Gefühle, Empfindungen, Bewertungen, Zufriedenheiten und andere subjektive Einschätzungen bei Kindern in gleichem Maße möglich und erfolgreich ist, da solche Daten von grundsätzlich anderer Qualität sind als objektive Angaben. Sie sind z. B. nicht direkt an objektiven Außenkritetien auf ihre Richtigkeit hin überprüfbar, und sie verlangen interne Bewertungen und Urteile. In der Literatur ist diese Frage umstritten. Im Kindersurvey wurde sie praktisch durch die Aufnahme und Auswertung solcher Fragen bejaht. Auch bei diesen Fragen läßt sich teilweise prüfen, inwieweit
Kinder- und Elternangaben übereinstimmen. Dabei zeigt sich, daß die Übereinstimmung hinsichtlich subjektiver Bewertungen, Einstellungen und Empfindungen deutlich geringer ausfällt, was aber durchaus plausibel ist. Subjektive Urteile unterliegen den persönlichen Wertungen und Meinungen der Befragten, und unterschiedliche Beurteilungsergebnisse von Kindern und Eltern sind somit verständlich. Sie sprechen unseres Erachtens für die Entwicklung eigenständiger Urteilskriterien bei Kindern und nicht für deren Unfähigkeit, Auskünfte über sich selbst zu erteilen. Die teilweise geringen Übereinstimmungen zwischen Kindern und Eltern bei diesen Angaben scheinen uns deshalb eher ein Argument für als gegen die Befragung von Kindern zu sein.

\section{Literatur}

Bauer, A. (unter Mitarbeit von Langenheim, H. / Schork, B.), 1984: Kinder als Informanten? Eine empirische Untersuchung über die Zuverlässigkeit der Schichteinstufung der Eltern durch Schüler aus der 4. Klasse. In: Meulemann, H./Reuband, K. H. (Hrsg.): Soziale Realität im Interview. Frankfurt: Campus.

Cohen, R. S./Orum, A. M., 1972: Parent-child consensus on socio-economic data obtained from sample surveys. Public Opinion Quarterly 36: 95-98.

Götte, R., 1979: Meßinstrumente zur Erfassung der häuslichen Lernumwelt von Kindern. Zeitschrift für empirische Pädagogik 3: 95-120.

Hess, R. D./Torney, J. V., 1967: The development of political attitudes in children. Chicago: Aldine.

ISCO, 1971: Internationale Standardklassifikation der Berufe. Statistisches Bundesamt Wiesbaden (Hrsg.), Systematische Verzeichnisse. Stuttgart.

Kaufmann, F. X./Lüscher, K., 1979: Wir brauchen eine Politik für Kinder. Neue Sammlung 19: 222-233.

Lüscher, K., 1977: Sozialpolitik für das Kind. Kölner Zeitschrift für Soziologie und Sozialpsychologie Sonderheft 19: Soziologie und Sozialpolitik: 591-628.

Meulemann, H./Wieken-Mayser, M., 1984: Kategorien der Sozialstatistik und Alltag der Familie. Die Übereinstimmung von Gymnasiasten des 10. Schuljahres mit ihren Eltern in Angaben zur Struktur und zur sozialen Position der Familie. In: Meulemann, H. Reuband, K.-H. (Hrsg.): Soziale Realität im Interview. Frankfurt: Campus.

Saint John, N., 1970: The validity of children's reports of their parents' educational level: A methodological note. Sociology of Education 43: 255-269. 